\title{
The Correlation of $\mathrm{C}$ - reactive protein And Phosphate Levels in Cord Blood Samples of Neonates with and without Sepsis
}

\author{
Rupita Kulshrestha ${ }^{1}$, Manish Raj Kulshrestha ${ }^{2 *}$, Rakesh Kumar Kalra ${ }^{3}$ \\ ${ }^{1}$ Assistant Professor, Department of Obstetrics and Gynecology, Mayo Institute of Medical Sciences, Barabanki, UP, India \\ ${ }^{2}$ Associate Professor, Department of Biochemistry Dr. Ram Manohar Lohia Institute of Medical Sciences, Lucknow, UP, India \\ ${ }^{3}$ Associate Professor, Department of Pediatrics, N C Medical College, Israna, Panipat, Haryana, India
}

\begin{abstract}
DOI: $10.36348 /$ sijog.2020.v03i03.001
| Received: 22.02.2020 | Accepted: 01.03.2020 | Published: 07.03.2020
\end{abstract}

*Corresponding author: Dr. Manish Raj Kulshrestha

\section{Abstract}

Introduction: Sepsis is one of the most common causes of morbidity and mortality in newborns, causing about $46 \%$ of global under-five child mortality. In sepsis, C - reactive protein (CRP) is elevated and Phosphate levels are low in blood and severity of hypophosphatemia also relates with poor outcome. The present study aims to measure levels of CRP, calcium $(\mathrm{Ca})$, phosphate $(\mathrm{P})$ and $\mathrm{Ca}: \mathrm{P}$ ratio in cord blood of neonates with or without sepsis. Methods: Cord blood samples of 60 neonates were enrolled for the study. Samples were divided into two groups on the basis of presence or absence of sepsis in first $24 \mathrm{hrs}$ of birth as decided by pediatrician. Measurement of CRP, calcium, phosphate, Calcium: phosphate ratio (Ca:P ratio) was done. Data so collected was analyzed with SPSS 20 software. Results: Neonates with sepsis had significantly high CRP and low phosphate levels in the cord blood. Ca:P ratio showed significant correlation with increasing CRP levels. Factors which did not show any significant correlation between the two groups were serum calcium levels, gestational age, birth weight and mode of delivery. $81.8 \%$ of neonates in group 2 (sepsis group) had phosphate level $<8.8 \mathrm{mg} / \mathrm{dl}$ (median level). Conclusion: Elevated CRP levels and Hypophosphatemia is present in neonates with sepsis. There is a negative correlation between serum phosphate and CRP levels. CRP has also been proposed as a diagnostic tool being easily measurable, affordable and convenient. Decreased Ca:P ratio should be taken care of while dealing with parenteral nutrition in such neonates. Phosphate levels must be carefully monitored in such cases.

Keywords: Cord blood C Reactive protein (CRP), hypophosphatemia, early onset neonatal sepsis (EONS), Ca:P Ratio.

Copyright @ 2020: This is an open-access article distributed under the terms of the Creative Commons Attribution license which permits unrestricted use, distribution, and reproduction in any medium for non-commercial use (NonCommercial, or CC-BY-NC) provided the original author and source are credited.

\section{INTRODUCTION}

Early onset neonatal sepsis (EOS) causes significant neonatal mortality, which accounts for $46 \%$ of global fewer than five child mortality [1]. Sepsis was the major reason for mortality in Neonatal Surgical ICU [2]. It is one of the most common causes of morbidity and mortality in newborns. Severe neonatal infections are one of the most significant causes of pediatric mortality, resulting in more than 500,000 deaths each year [3].

However, the relationship of hypophosphatemia with sepsis in pediatric patients is sparsely studied in literature. The present study aims to analyze the association of CRP, phosphate levels and $\mathrm{Ca}$ : $\mathrm{P}$ ratio with sepsis in neonates.

\section{MATERIALS AND METHODS}

This study was conducted at a tertiary care centre in northern India. After taking consent from parents/ mother, the cord blood samples of 60 neonates were collected at birth as study samples randomly. They were than divided into two groups as group-1 neonates without sepsis and group- 2 neonates with sepsis as decided by the treating pediatrician on the basis of development of features like refusal to take feeds, lethargy, irritability, poor cry, vomiting, diarrhea, hypothermia, cyanosis and fever within first 24 hours of birth. Suspicion/ presence of any structural malformations in the neonate was taken as exclusion criteria and allowed to clot for 1 hour. The serum was separated after centrifugation at $10000 \mathrm{rpm}$ for 10 
minutes and was analyzed for calcium, phosphorous and CRP within $24 \mathrm{hrs}$ of collection. The calcium and phosphorous were measured using arsenazo method and phosphomolybate blue method respectively by kits from Roche diagnostics. CRP was analyzed on Erbachem using immunoturbidimetric tecnique by kit from Agappe diagnostics. The calcium: phosphorous ratio (Ca:P ratio) was calculated. The data was analyzed on SPSS 20 software.

\section{RESULTS}

Our study showed that cord blood CRP levels in neonates with sepsis were higher than neonates without sepsis. Neonates with sepsis had significantly low phosphate levels in the cord blood suggesting that hypophosphatemia is associated with presence of sepsis. Levels of serum calcium did not show any significant difference in the two groups however $\mathrm{Ca}: \mathrm{P}$ ratio showed significant correlation with increasing CRP levels $\quad\left(r^{2}=0.269 ; \quad p=0.001\right)$ on Pearson correlation. Factors of gestational age, birth weight and mode of delivery did not show any significant correlation ( $p>0.005) 81.8 \%$ of neonates in group 2 (with sepsis) had phosphorous below the median value $(8.8 \mathrm{mg} / \mathrm{dl})$. The CRP was negatively correlated with phosphorous levels $(\mathrm{r}=-0.373 ; \mathrm{p}=0.018)$.

Table-1: Comparison of different variables amongst group $1 \&$ group 2

\begin{tabular}{|c|c|c|c|}
\hline & $\begin{array}{l}\text { Group } 1 \\
\text { (n=30,without sepsis) } \\
\text { (Mean } \pm \text { S.D.) }\end{array}$ & $\begin{array}{l}\text { Group } 2 \\
\text { (n=30, with sepsis) } \\
\text { (Mean } \pm \text { S.D.) }\end{array}$ & p Value \\
\hline Gestation age(weeks) & $38.2 \pm 1.46(35.34-42)$ & $36.5 \pm 1.53$ & 0.653 \\
\hline Birth weight (grams) & $3280(1800-3400)$ & $2650(1700-3400)$ & 0.273 \\
\hline CRP (mg/L) & $0.55 \pm 0.18$ & $1.34 \pm 0.55$ & $<0.001$ \\
\hline Calcium (mg/dl) & $10.76 \pm 1.79$ & $10.63 \pm 1.75$ & 0.820 \\
\hline Phosphorous (mg/dl) & $12.51 \pm 7.71$ & $8.33 \pm 4.48$ & 0.038 \\
\hline Ca:P ratio & $1.13 \pm 0.57$ & $1.67 \pm 0.74$ & 0.043 \\
\hline
\end{tabular}

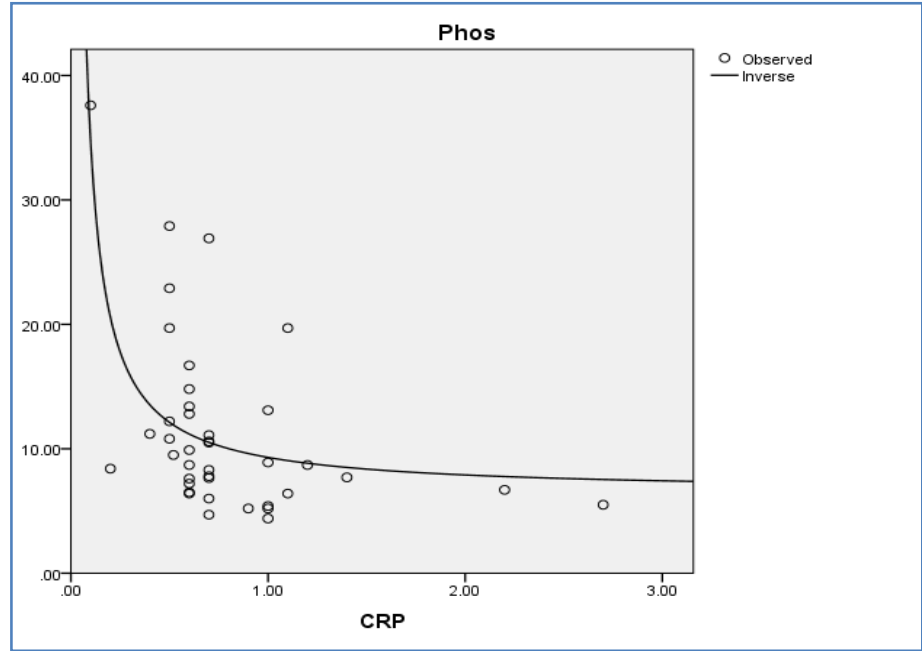

Fig-1: The correlation of cord blood CRP levels with cord blood phosphorous levels

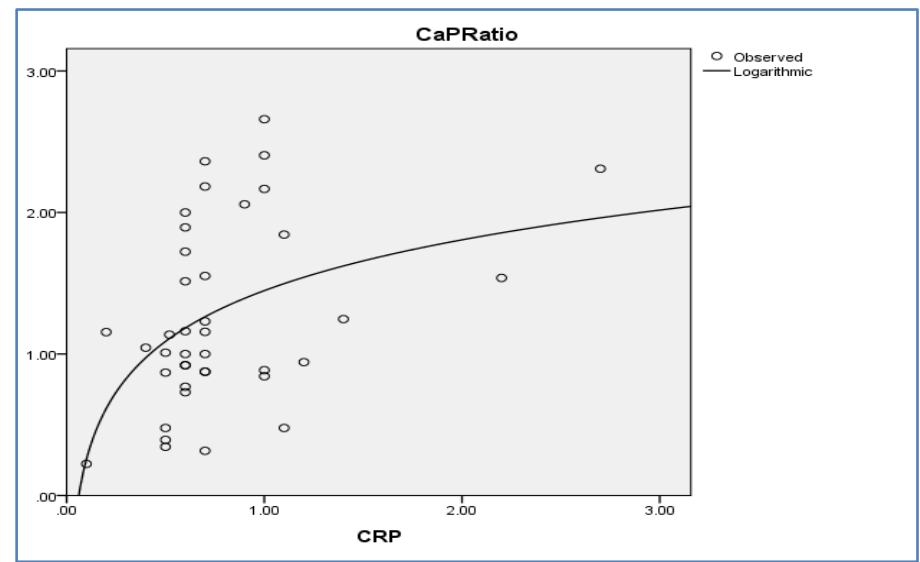

Fig-2: The correlation of cord blood CRP levels with cord blood calcium: phosphorous ratio 


\section{DISCUSSION}

The exact cause of hypophosphatemia in patients with infections has not yet been fully understood. Hypophosphatemia is a metabolic disturbance with serious complications. Phosphate ion are important for normal bone mineralization, and is involved in other biological processes like adenosine triphosphate (ATP) production, signal transduction, nucleotide metabolism, enzyme regulation etc.

Various authors have studied the association of sepsis with hypophosphatemia in adults like, low serum phosphate levels were observed in $24-80 \%$ in patients with early sepsis, $22-45 \%$ of cases with pneumonia and $42-65 \%$ of patients with bacterial infection in general [4]. Few authors have reported hypophosphatemia in patients with sepsis having prevalence of $22-80 \%$ and in some case it has correlated with the severity of illness and patient's outcome as well [4]. Shor $\mathrm{R}$ et al. studied the association of hypophosphatemia with mortality in adults and have concluded that severe hypophosphatemia is associated with 8 fold increase in mortality risk and even hypothesized that it can serve as an independent indicator of mortality in sepsis [5]. Prolonged stay in PICU and worse outcome were associated with hypophosphatemia as studied by Rady [6]. Barak et al. demonstrated an association of hypophosphatemia with high levels of Tumor Necrosis Factor- $\alpha$ (TNF- $\alpha$ ) and Interleukin-6 (IL-6) in adult patients with sepsis [7].

C-reactive protein (CRP) is a well-known indicator of inflammation, which is an acute phase protein, synthesized by hepatocytes due to stimulatory effect of the inflammatory cytokines such as IL-6, IL-1, TNF- $\alpha$ etc, the concentration of which rises during infection before CRP by 24-48 hours [7]. Level of hypophosphatemia correlated with high levels of inflammatory cytokines and therefore, inverse relationship between CRP and Phosphate could be hypothesized [7]. Injection of IL-6, TNF $\alpha$ and IL-1 $\beta \mathrm{m}$ when injected in mice markedly decreased the phosphate serum levels. A correlation between the high inflammatory cytokines levels and hypophosphatemia during early sepsis was established by Barak et al. [7] hence, they suggested inclusion of cytokine levels and hypophosphatemia in sepsis evaluation and prognosis.

In our study also, $81.8 \%$ of neonates in group 2 (with sepsis) had phosphorous below the median value $(8.8 \mathrm{mg} / \mathrm{dl})$. Haglin et al. analysed relationship between CRP and phosphate levels in adult patients with infection and reported a higher mean CRP value and hypophosphatemia in patients with sepsis [8] similar to our findings in neonates.

Mathai E et al. concluded that in comparison to leukocyte counts and ratios, CRP levels at 24 hour proved to be the single best indicator for diagnosing
EOS (Early Onset Sepsis).When utilized with caution, CRP could help in reducing antimicrobial use in the neonates [9].

Nowadays, using automated analyzers quantification of CRP at lower levels is also possible. Ishibashi et al. proposed that CRP may have diagnostic value in neonatal infection as in neonates without sepsis very low CRP concentrations are found in the cord blood and sera in the immediate postnatal period [10].

Kheir $\mathrm{AE}$ et al. studied the role of C-reactive protein in the diagnosis of neonatal sepsis, and examine the correlation between C-reactive protein, blood culture and risk factors for sepsis [3]. The correlation between CRP and birth weight and gestational age was not significant. Belachew A et al. assessed the prevalence of neonatal sepsis and its association with birth weight and gestational age among admitted neonates in Ethiopia they also failed to establish any significant relation between neonatal sepsis and gestational age or birth weight [11]. In our study also we did not find any significant association between gestational age and birth weight among the two groups.

Suryavanshi A studied the association of Creactive protein with maternal chorioamnionitis in premature rupture of membranes (PROM) and earlyonset neonatal sepsis (EONS)(12). Chorioamnionitis is defined by maternal fever, leukocytosis $(>15,000$ white blood cells $[\mathrm{WBCs}] / \mathrm{mm} 3)$, maternal tachycardia, uterine tenderness, foul odor of amniotic fluid, and fetal tachycardia at delivery, is also a major risk factor for neonatal sepsis [12]. They concluded that mothers with premature leaking have higher maternal blood CRP levels and their babies had higher risk of developing EONS. Such cases must be delivered early and the neonate should undergo and neonatal screening for EONS [12]. In the present study we have taken cord blood sample of baby during delivery. The rise of CRP in our samples signifies EONS as also observed by Mithal et al. [13]

Significantly high C-reactive protein levels and low sodium levels were observed during hypophosphatemia episodes in severely disabled individuals with neurological disorders as observed by Saito at al [14].

Chiesa et al. analyzed serum CRP concentrations in 148 healthy babies and tried to establish the upper limits of normal for CRP in the immediate postnatal period and they found that only 19 $(13 \%), 54(36 \%)$, and $55(37 \%)$ of the neonates had detectable concentrations of CRP $(\sim 4 \mathrm{mg} / \mathrm{L})$ at birth (cord blood), at $24 \mathrm{~h}$, and at $48 \mathrm{~h}$, respectively $[15,16]$.

Some authors hypothesize that the immature liver function of premature infants decreases their 
ability to generate and secrete CRP into the serum and/or there may be decreased ability of CRP to actually bind to phosphocholine and activate the complement system [17]. This complement activation may lead to hypophosphatemia or intracellular shift of phosphorous (as apparent in proven sepsis). Our study shows decline in phosphate levels with increase in CRP. A higher incidence of hypophosphatemia was reported during the initial $24 \mathrm{hr}$ of hospitalizations; phosphate levels $<2 \mathrm{mg} / \mathrm{dl}$ were especially in cases of gram negative sepsis [5].

Vitamin D, a negative acute phase reactant, is decreased in acute inflammation. It may increase serum phosphorous levels by decreasing its urinary excretion. Also, in chronic renal failure adults, the level of the inflammatory parameters CRP and IL-6 was significantly higher even after adjustment for age, gender and eGFR. Moreover, serum phosphorous concentration was positive, significant, and independently associated with CRP and IL-6, and it was an independent risk factor for the presence of an inflammatory state. However current study does not comment upon any such factor as we have taken only cord blood sample of neonates for the study.

The provision of parenteral calcium and phosphorus in a 1.7:1 ratio have been resulting in a balanced retention of both minerals in comparison to higher or lower ratio. The use of this calcium/phosphorus ratio ( $\mathrm{Ca}: \mathrm{P}$ ratio) appears to be appropriate for the preterm infant receiving total parenteral nutrition [18]. Thus, it may be essential to find out the impact of CRP levels on serum phosphorous levels and calcium: phosphorous ratio. It may be helpful in adjustment of calcium: phosphorous ratio in parenteral administration to achieve optimum absorption of these minerals in neonates. Altered Cap ratio in sepsis suggests that this has to be taken care while planning parenteral nutrition for such neonates.

\section{CONCLUSION}

Hypophosphatemia is present in patients with acute infectious diseases. Sepsis is an inflammatory process involving certain cytokines and there is a negative correlation between serum phosphate and CRP levels. Similar to our results, low serum phosphate levels are associated with an increase in CRP concentration as also studied by other authors [3, 4, 6-8, 14]. CRP has also been proposed as a diagnostic tool. Normalization of CRP elevation appears to be helpful in determining the response to antimicrobial therapy and duration of treatment [3]. Health care providers should adhere to aseptic precautions while performing procedures, especially in preterm and low birth weight infants [11]. A single CRP level done at the time of a neonatal sepsis evaluation can guide about the continuance of antibiotic therapy. American Academy of Pediatrics Committee on Fetus and Newborn recommends that bacterial sepsis is unlikely with normal CRP levels and antibacterial agents may be discontinued [19].

High cost of other inflammatory markers restricts their routine application in low resource settings and CRP being easily measurable, more affordable and convenient to use is a good marker for the diagnosis of neonatal sepsis especially in developing communities with limited resources. To conclude, C-reactive protein is a cheap and reliable marker of neonatal sepsis, especially in developing countries with low resource settings [3]. Ca:P ratio which is found to significantly associated with neonatal sepsis [17], this factor should be kept in mind while planning parenteral nutrition in such neonates. Since severe hypophosphatemia is related with high morbidity and mortality [5], so it has to be diagnosed and treated vigorously when encountered.

\section{REFERENCES}

1. Khalil, N., Blunt, H. B., Li, Z., \& Hartman, T. (2020). Neonatal early onset sepsis in Middle Eastern countries: a systematic review. Archives of Disease in Childhood.

2. Siddharth, V., Gupta, S. K., Agarwala, S., Satpathy, S., \& Goel, P. (2019). Outcome of care provided in neonatal surgery intensive care unit of a public sector tertiary care teaching hospital of India. Journal of Indian Association of Pediatric Surgeons, 24(4), 257.

3. Kheir, A. E., Jobara, G. A., Elhag, K. M., \& Karar, M. Z. (2013). Qualitative C-reactive protein as a marker of neonatal sepsis in a tertiary neonatal unit in Sudan. Healthcare in Low-resource Settings, 1(2), e21-e21.

4. Antachopoulos, C., Margeli, A., Giannaki, M., Bakoula, C., Liakopoulou, T., \& Papassotiriou, I. (2002). Transient hypophosphataemia associated with acute infectious disease in paediatric patients. Scandinavian journal of infectious diseases, 34(11), 836-839.

5. Shor, R., Halabe, A., Rishver, S., Tilis, Y., Matas, Z., Fux, A., \& Weinstein, J. (2006). Severe hypophosphatemia in sepsis as a mortality predictor. Annals of Clinical \& Laboratory Science, 36(1), 67-72.

6. Rady, H. I., Abdel, K., \& Mohamed, K. (2014). Prevalence and risk factors of hypophosphatemia in pediatric intensive care unit. J Anesth Crit Care Open Access, 1, 00033.

7. Barak, V., Schwartz, A., Kalickman, I., Nisman, B., Gurman, G., \& Shoenfeld, Y. (1998). Prevalence of hypophosphatemia in sepsis and infection: the role of cytokines. The American journal of medicine, 104(1), 40-47.

8. Håglin, L., Burman, L. A., \& Nilsson, M. (1999). High prevalence of hypophosphataemia amongst patients with infectious diseases. A retrospective study. Journal of internal medicine, 246(1), 45-52. 
9. Mathai, E., Christopher, U., Mathai, M., Jana, A. K., Rose, D., \& Bergstrom, S. (2004). Is Creactive protein level useful in differentiating infected from uninfected neonates among those at risk of infection. Indian Pediatr, 41(9), 895900.

10. Ishibashi, M., Takemura, Y., Ishida, H., Watanabe, K., \& Kawai, T. (2002). C-reactive protein kinetics in newborns: application of a high-sensitivity analytic method in its determination. Clinical chemistry, 48(7), 11031106.

11. Belachew, A., \& Tewabe, T. (2020). Neonatal sepsis and its association with birth weight and gestational age among admitted neonates in Ethiopia: systematic review and metaanalysis. BMC pediatrics, 20(1), 55.

12. Suryavanshi, A., \& Kalra, R. (2019). Study of association of $\mathrm{C}$-reactive protein with maternal chorioamnionitis and early-onset neonatal sepsis in premature rupture of membranes deliveries: A diagnostic dilemma. International Journal of Applied and Basic Medical Research, 9(4), 236.

14. Mithal, L. B., Palac, H. L., Yogev, R., Ernst, L. M., \& Mestan, K. K. (2017). Cord blood acute phase reactants predict early onset neonatal sepsis in preterm infants. PLOS one, 12(1).

15. Saito, Y., Aoki, Y., Takeshita, E., Saito, T., Sugai, K., Komaki, H., ... \& Sasaki, M. (2014). Hypophosphatemia is a common complication in severely disabled individuals with neurological disorders and is caused by infection, refeeding and Fanconi syndrome. Brain and Development, 36(10), 878-883.

16. Chiesa, C., Signore, F., Assumma, M., Buffone, E., Tramontozzi, P., Osborn, J. F., \& Pacifico, L. (2001). Serial measurements of C-reactive protein and interleukin- 6 in the immediate postnatal period: reference intervals and analysis of maternal and perinatal confounders. Clinical chemistry,47(6), 10161022.

17. Schanler, R. J., \& Rifka, M. (1994). Calcium, phosphorus and magnesium needs for the lowbirth-weight infant. Acta paediatrica (Oslo, Norway: 1992). Supplement, 405, 111-116.

18. Simonsen, K. A., Anderson-Berry, A. L., Delair, S. F., \& Davies, H. D. (2014). Earlyonset neonatal sepsis. Clinical microbiology reviews, 27(1), 21-47

19. Pelegano, J. F., Rowe, J. C., Carey, D. E., LaBarre, D. J., Raye, J. R., Edgren, K. W., \& Horak, E. (1989). Simultaneous infusion of calcium and phosphorus in parenteral nutrition for premature infants: use of physiologic calcium/phosphorus ratio. The Journal of pediatrics, 114(1), 115-119.

20. Khalak, R., Malhotra, A., \& Santos, R. P. (2018). Use of a single C-reactive protein level in decision-making during neonatal sepsis evaluation. Journal of Clinical Neonatology, 7(3), 146. 